\title{
Sex reversal linked to Sox3
}

Sex in mammals is genetically determined; females normally have two $\mathrm{X}$ chromosomes, and males normally have one $\mathrm{X}$ and one $\mathrm{Y}$ chromosome. But sometimes an individual with two $\mathrm{X}$ chromosomes is born with male genitalia, in a case of XX male sex reversal. The incidence of XX male sex reversal in humans is roughly 1 in 20,000-25,000 male births. Results from recent studies suggest that changes in expression of the gene Sox 3 can cause complete XX male sex reversal in mice and may underlie some cases of unexplained $\mathrm{XX}$ male sex reversal in humans.

Male development is normally dependent on early embryonic expression of the gene Sry, located on the Y chromosome, which induces testis formation and activates the male development pathway. But Paul Thomas (University of Adelaide, Australia) and his colleagues found that ectopic expression of Sox3, a gene located on the $\mathrm{X}$ chromosome, in developing gonads resulted in XX mice that were male in appearance, reproductive structures and behavior but were sterile

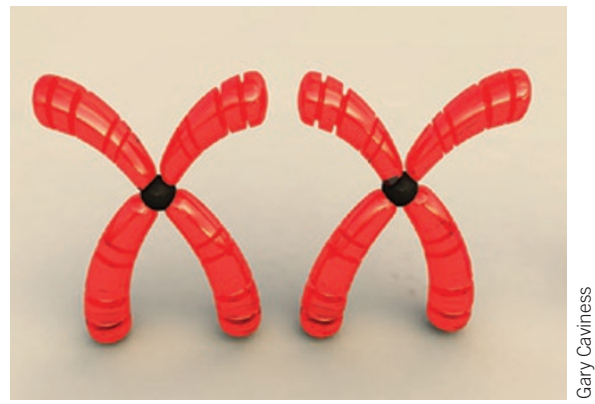

owing to a lack of sperm production (J. Clin. Invest. doi:10.1172/JCI42580; published online 22 December 2010).

Sox3 is important in the development of the brain and central nervous system but was not previously known to be able to trigger male development. The new results from Thomas' studies indicate that Sox3 can act as a surrogate for Sry and shed light on the evolutionary relationship between these two genes. "We have suspected for a long time that SOX3 is the evolutionary precursor gene for SRY. By showing that
SOX3 can activate the male pathway in the same way as SRY, we now believe this to be true," said Thomas in a press release.

Given that Sox 3 ectopic expression was able to induce sex reversal in mice, it seemed possible that changes to $S O X 3$ could be associated with XX male sex reversal in humans. Therefore, in collaboration with researchers at other institutions in Australia and internationally in London (UK), Lisbon (Portugal) and Los Angeles and Berkeley (CA), Thomas' group analyzed the genomes of 16 humans with XX male sex reversal and found that 3 of these individuals had genomic rearrangements near the SOX3 locus. Such rearrangements may be the underlying cause of sex reversal in some affected individuals. In a press release, Thomas stated, "This discovery provides new insight into the genetic causes of disorders of sexual development... [and will] ultimately help us to develop therapies or technologies to improve clinical outcomes."

\section{Monica Harrington}

\section{RESTORING MEMORY USING PROTEIN}

Increasing the levels of a memory-related protein in the brains of mice with symptoms of Alzheimer's disease ameliorated the learning and memory deficits of these mice, researchers recently reported. These results point to a potential approach for treating people with Alzheimer's disease.

In humans, Alzheimer's disease is characterized by the accumulation of amyloid plaques, formed mainly of the amyloid- $\beta$ peptide, and neurofibrillary tangles, which consist of the protein tau. Amyloid- $\beta$ accumulation has a key role in the development of the cognitive deficits characteristic of Alzheimer's disease in humans. While much previous research has focused on finding ways to remove accumulated amyloid- $\beta$ or tau, this research team looked further downstream.

In their study, Salvatore Oddo and colleagues from the University of Texas Health Science Center in San Antonio used 6-monthold transgenic mice (Proc. Natl. Acad. Sci. USA doi:10.1073/pnas.1012851108; published online 13 December 2010) that are commonly used as an animal model of Alzheimer's. These mice develop amyloid- $\beta$ and tau accumulation. Oddo and his team found that amyloid- $\beta$ accumulation interfered with the activity of the CAMP response element-binding protein (CREB), which is involved in memory and learning.

The researchers injected brains of transgenic and non-transgenic mice with either a virus expressing CREB-binding protein (CBP), which activates CREB, or a sham injection. Seven days later, the researchers started training the mice on a maze used to study spatial memory and learning. Transgenic mice that had received CBP performed as well as healthy mice that had received either CBP or a sham injection, whereas transgenic mice that had received the sham injection had trouble learning and remembering. These results show that overexpressing CBP in the brains of the transgenic mice (through the viral injection of CBP) reversed spatial learning and memory deficits in these animals.

Further analyses showed that CBP injections restored CREB activity and memory and learning ability without impacting the levels of amyloid- $\beta$ or tau. Additionally, injecting CBP into transgenic mice led to increased levels of a protein called brain-derived neurotropic factor (BDNF), which is involved in memory formation. CREB facilitates the transcription of the gene encoding BDNF.

The authors conclude that their results show that cognitive dysfunction in a mouse model of Alzheimer's disease can be ameliorated without affecting the amyloid- $\beta$ or tau pathology. They note that CBP delivery may also be beneficial for treating other neurodegenerative diseases, such as Huntington's disease and Rubinstein-Taybi syndrome, in which CBP dysfunction has been reported. Kirsten Dorans 